\title{
Noise and Intermodulation Properties of Tunable Recursive Active MMIC Filters for Future Adaptive On-chip Radar Receivers
}

\author{
Robert Malmqvist $\dagger$, Andreas Gustafsson $\dagger$, Magnus Danestig $\dagger^{*}$, Aziz Ouacha $\dagger$, \\ Sven Hagelin $\dagger$, and Staffan Rudner $\dagger$
}

\author{
†FOA Defense Research Establishment, Linköping, Sweden, \\ rma@lin.foa.se andreasg@lin.foa.se magnus.danestig@acreo.se aziz@lin.foa.se svehag@lin.foa.se starud@lin.foa.se
}

\begin{abstract}
The possibilities and the limitations of tunable narrow band recursive active MMIC filters with respect to future adaptive on-chip radar receivers are investigated. We compare the measured and the simulated results of two recursive active MMIC filters with the corresponding theoretical results obtained using our analytical filter models. A good agreement between these results is generally found. Using our analytical filter models we also estimate the noise and intermodulation properties of the low noise amplifier used in the filters that are necessary in order to achieve a filter performance adequate for future adaptive on-chip radar receivers.
\end{abstract}

\section{INTRODUCTION}

Methods to decrease the vulnerability of jamming from unwanted signals are of prime importance in modern radar systems. A reduction of size and cost of the multi-channel microwave receivers planned for future advanced adaptive radar antennas is also becoming increasingly important [1]. As a consequence of the wish to reduce receiver size and complexity, increased interest has been focused on the realisation of active monolithic microwave integrated circuit (MMIC) filters. Such filters are very attractive since they may potentially be used to replace the passive and bulky off-chip filters traditionally used in microwave receiver front-ends. A frequency tunable narrow-band active MMIC filter could also help to reduce the possibility of unwanted signals to reach the latter part of the receiver [2]. Such a bandpass filter must however meet very stringent requirements in terms of a sufficiently low filter noise figure in combination with a sufficiently high filter gain and filter spurious-free dynamic range $(S F D R)$ respectively. Typical requirements for a tunable active filter used in the microwave receiver for an adaptive radar can be found in [3] and are summarised in Table I (an SFDR of $113 \mathrm{~dB} / \mathrm{Hz}^{2 / 3}$ is equivalent to an $S F D R$ of about $64 \mathrm{~dB}$ for a receiver bandwidth $(B W)$ of $18 \mathrm{MHz})$. The recursive active MMIC filter [4] is interesting for narrow-band and low-noise applications since high-Q filters of this type can be designed with a high filter gain in combination with a filter noise figure approaching that of the low-noise amplifier (LNA) used in the filters [5]. In an analytical study of the optimum filter noise figure [6], this filter type was shown to be promising for receiver front-ends where it could potentially replace the classical cascade of an LNA and a passive filter. Analytical studies of the third order intercept point and the optimum filter noise figure for two different recursive active MMIC filter topologies as a function of filter Q-value were first presented in [7], [8] and [9] respectively. Starting from the requirements of typical microwave receivers for future adaptive radars we investigate in this paper both theoretically and experimentally the possibilities and the limitations of tunable narrow band recursive active MMIC filters with respect to such on-chip radar receivers. Measurement results together with simulations using HP EEsof CAD tool Libra are presented for two different recursive active MMIC filter designs. These results are compared with the theoretical results from our published analytical filter models [7], [8] and [9].

\section{TABLE I}

Typical requirements for a tunable active filter used in microwave receivers for adaptive radar (X-band).

\begin{tabular}{|ll|}
\hline Agile frequency band & $8-10 \mathrm{GHz}$ \\
Relative $20 \mathrm{~dB}$ bandwidth $\left(B_{20 \mathrm{~dB}}\right)$ & $10 \%$ \\
Center frequency gain $(\mathrm{G})$ & $>10 \mathrm{~dB}(<25 \mathrm{~dB})$ \\
Noise figure $(N F)$ & $<5 \mathrm{~dB}$ \\
Input third order intercept point $\left(I I P_{3}\right)$ & $\geq 0 \mathrm{dBm}$ \\
Spurious-free dynamic range $(S F D R)$ & $113 \mathrm{~dB} / \mathrm{Hz}^{2 / 3}$
\end{tabular}

\section{DEFINITIONS}

A schematic model of a recursive active filter with the amplifier placed in a feed-forward arrangement between two passive couplers is depicted in Fig. 1.

In order to simplify the filter analysis, all elements are assumed to be matched to $50 \Omega$. The amplifier is modelled by an ideal gain-block with gain $G_{A}$, a third

\footnotetext{
* Presently at ACREO AB, S-602 21, Norrköping, Sweden
} 
order output intercept point $O I P_{3, A}$ and a noise figure $N F_{A}$. The two passive couplers are assumed to be lossless and perfectly linear with coupling factors $\alpha_{i}, \beta_{i}$ $(i=1,2)$. A constant delay $\tau$ corresponds to the total loop time delay in a real filter circuit. Figure 1 shows that the filter loop gain $V$ can be expressed as $V=G_{A} \beta_{1} \beta_{2}(V$ $<1)$ and that $\tau$ can be related to the center frequency $\omega_{c}$ as a multiple (i.e. in terms of an integer $n$ ) of $2 \pi$.

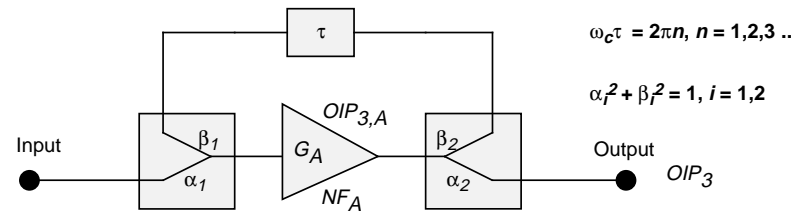

Fig. 1. Schematic model of a recursive active filter.

\section{MESAUREMENTS, SIMULATIONS AND ANALYTICAL RESULTS}

We have compared the measurement and simulation results of two different tunable recursive active MMIC filters designed for the X-band with the corresponding theoretical results obtained from our analytical filter models [7, 8 and 9]. The filters were fabricated by Philips Microwave Limeil using their $0.2 \mu \mathrm{m}$ GaAs/AlGaAs PHEMT based MMIC process. They were designed using the novel concept of self-switched time shifters for the filter center frequency tuning [10]. One of the filter designs, denoted here as the Medium- $Q$ filter, was first presented and evaluated in [10]. This filter design consists in addition to a three-bit time shifter of two lumped and approximately symmetrical couplers together with an LNA. The other filter design, which we denote as the Low-noise filter, differs only in the design of the LNA and in the use of a lumped unsymmetrical input coupler in order to minimise the filter noise figure [5]. Photos of the layouts of the two MMIC filter designs are shown in Fig. 2 and Fig. 3 respectively.

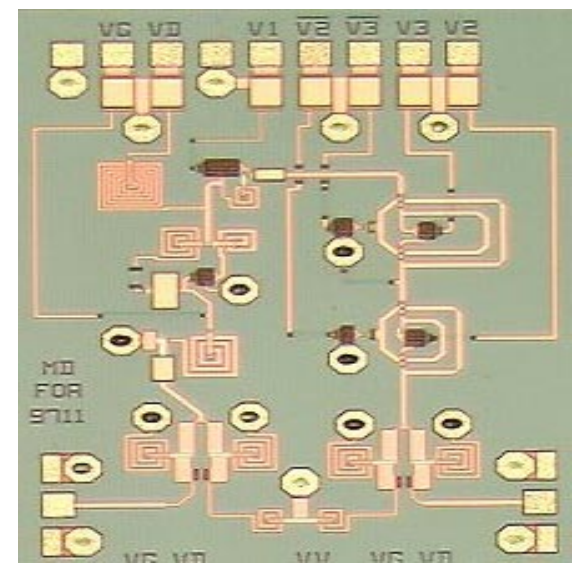

Fig. 2. Photo of a tunable recursive active MMIC filter with symmetrical input/output couplers (Medium- $Q$ filter). Chip size: $1.6 \times 2.1 \mathrm{~mm}^{2}$.

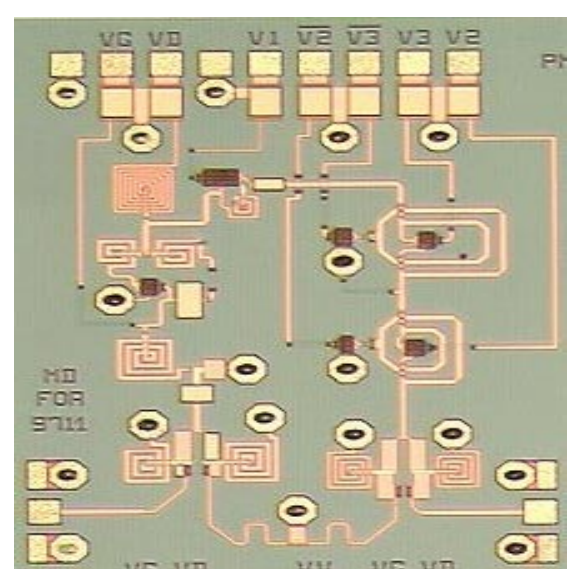

Fig. 3. Photo of a tunable recursive active MMIC filter with an unsymmetrical input coupler (Low-noise filter). Chip size: $1.6 \times 2.1 \mathrm{~mm}^{2}$.

Table II shows for each of the two filter designs, a comparison between the measured results, the simulation results (using the HP EEsof CAD tool Libra) and the theoretical results from our analytical filter models.

TABLE II

Q-value, center frequency gain, noise figure, third order output intercept point and spurious-free dynamic range $(B W=18 \mathrm{MHz})$ at $f_{c}=8.7 \mathrm{GHz}$ for the Medium- $Q$ filter and the Low-noise filter respectively ( $\tau=2 / f_{c}$, i.e. $\left.n=2\right)$.

Medium-Q filter: $\left(I_{D S}=27 \mathrm{~mA}, V_{D}=3.0 \mathrm{~V}, \beta_{1}=-4.2 \mathrm{~dB}, \beta_{2}=\right.$ $\left.-3.9 \mathrm{~dB}, G_{A}=5.5 \mathrm{~dB}, N F_{A}=3.5 \mathrm{~dB}, O P_{3, A}=9.3 \mathrm{dBm}\right)$.

Low-noise filter: $\left(I_{D S}=30 \mathrm{~mA}, V_{D}=3.0 \mathrm{~V}, \beta_{1}=-9.3 \mathrm{~dB}, \beta_{2}=\right.$ $\left.-4.0 \mathrm{~dB}, G_{A}=7.6 \mathrm{~dB}, N F_{A}=3.0 \mathrm{~dB}, O I P_{3, A}=12.3 \mathrm{dBm}\right)$.

\begin{tabular}{lccccc}
$\begin{array}{l}\text { Medium- } \\
Q\end{array}$ & $\boldsymbol{Q}$ & $\begin{array}{c}\boldsymbol{G} \\
{[\mathrm{dB}]}\end{array}$ & $\begin{array}{c}\boldsymbol{N F} \\
{[\mathrm{dB}]}\end{array}$ & $\begin{array}{c}\boldsymbol{O I P 3} \\
{[\mathrm{dBm}]}\end{array}$ & $\begin{array}{c}\boldsymbol{S F D R} \\
{[\mathrm{dB}]}\end{array}$ \\
\hline Measured & 23 & 10.2 & 7.5 & 8 & 61.2 \\
Simulatd. & 23 & 10.2 & 6.9 & -0.5 & 55.9 \\
Model & 19 & 9.0 & $6.5^{*}$ & 0.0 & 57.3 \\
$\begin{array}{l}\text { Low- } \\
\text { noise }\end{array}$ & $\boldsymbol{Q}$ & $\boldsymbol{G}$ & $\mathbf{N F}$ & $\boldsymbol{O I P 3}$ & $\boldsymbol{S F D R}$ \\
\hline Measured & 11 & 7.6 & 6.0 & 8 & 63.9 \\
Simulatd. & 10 & 7.6 & 4.8 & 5.1 & 62.8 \\
$\quad$ & 9 & 8.0 & $4.1^{*}$ & 5.1 & 63.0 \\
Model & 9 & & & & \\
\hline
\end{tabular}

The measured and the simulated results of the $M e$ dium- $Q$ filter and Low-noise filter were obtained at a drain bias $\left(V_{D}\right)$ equal to $3.0 \mathrm{~V}$ and at a drain-source current $\left(I_{D S}\right)$ of $27 \mathrm{~mA}$ and $30 \mathrm{~mA}$ respectively. All three bits of the time shifter were biased in the same tuning state corresponding to a measured filter center frequency of $8.7 \mathrm{GHz}$. The measured results were obtained with a post production tuning of the gate bias $\left(V_{G}\right)$ in order to compensate for a deviation in filter gain due to process parameter variations. This did not affect the other pa- 
rameters compared. The loop time delay $\tau$ was chosen equal to $4 \pi / \omega_{\text {c }}$ (i.e. $n=2$ ). The values of $\beta_{1}, \beta_{2}, G_{A}$, $N F_{A}$ and $O I P_{3, A}$ used in our analytical filter models were found from simulations of the two filter designs.

It can be noted from Table II that the theoretical results from our analytical filter models are generally in a good agreement with the corresponding measured and simulated results of the two filters. The small discrepancy in the analytically modelled center frequency filter gain and filter Q-value compared with the corresponding measured and simulated results (see Table II) can mainly be explained by the fact that the analytical model is based on the assumption of perfectly impedance matched circuit elements. The somewhat lower value of the analytically modelled filter noise figure in comparison with the corresponding measured and simulated filter noise figure (see also Table $\mathrm{II}^{*}$ ) can to a large degree be explained by the fact that the effects on the filter noise figure of losses in a real passive coupler were neglected in this analysis. The total losses of the input coupler were estimated by simulations at about $1 \mathrm{~dB}$. Hence, a $0.5 \mathrm{~dB}$ higher value would be expected for the theoretically estimated values of the filter noise figure, if we assume that the total losses of the input coupler are uniformly distributed on each coupler leg [7]. Table II also shows that the values of the simulated and the analytically modelled $\mathrm{OIP}_{3}$ and $S F D R$ are somewhat lower than the corresponding measured values. This is most likely due to the fact that our analysis [8] was made assuming a worst case scenario; i.e. that the higher order harmonics always combine in phase and add constructively to the filter intermodulation product (thus resulting in a conservative value of $\mathrm{OIP}_{3}$ ). In a real filter circuit on the other hand, all the higher order harmonics will probably not combine in phase at all frequencies, thus resulting in a lower intermodulation product and therefore in a higher value of $\mathrm{OIP}_{3}$ and $S F D R$ respectively.

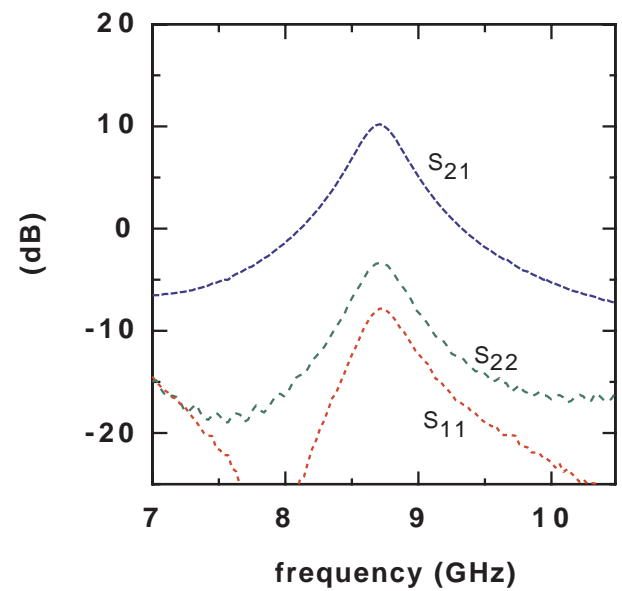

Fig. 4. Measured s-parameters for the Medium- $Q$ filter at the center frequency $8.7 \mathrm{GHz}$.

The measured filter s-parameters of the two filter designs at a center frequency $8.7 \mathrm{GHz}$ are shown in
Figs. 4 and 5 respectively. It can be noted that the filter selectivity in terms of $B_{20 d B}$, for example, is rather low. For the Medium- $Q$ filter, $B_{20 d B}$ is higher than 0.4 and the corresponding value for the Low-noise filter is even higher. Moreover, the measured filter noise figures (see Table II) are higher than the required noise figure of below $5 \mathrm{~dB}$ (see Table I). Hence, the filter performance of the two evaluated recursive active MMIC filter designs is not adequate for the microwave receivers in future adaptive radars. We have therefore used our validated analytical filter models to estimate the component characteristics necessary for such applications.

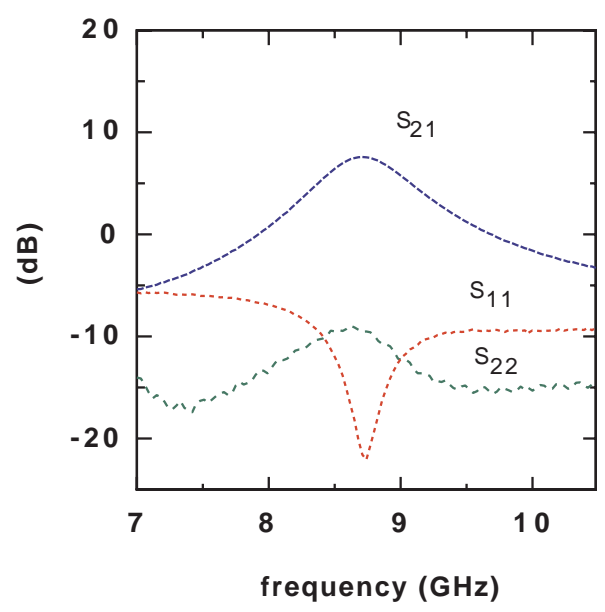

Fig. 5. Measured s-parameters for the Low-noise filter at the center frequency $8.7 \mathrm{GHz}$.

\section{ESTIMATED PERFORMANCE FOR OPTIMIZED RECURSIVE ACTIVE MMIC FILTERS}

In order to maximise the filter third order input intercept point $\left(I I P_{3}\right)$ the two couplers should be designed with values of $\beta_{1}$ and $\beta_{2}$ close to one [9]. However, according to an analytical evaluation of the optimum noise figure of recursive active MMIC filters [7], the coupler located at the input should be designed with a value of $\beta_{l}$ close to zero while the output coupler should be designed with $\beta_{2}$ equal to $V^{1 / 2}$ when we want to minimise the filter noise figure $N F$. Hence, if we choose to design the input coupler with a very low value of $\beta_{l}$ in order to minimise $N F$, the result is also a very low $I I P_{3}$. A filter designed in such a way; i.e. with a low $I I P_{3}$ and a low NF, (denoted here by index $a$ ) can however be connected in a cascade arrangement with a filter designed with a higher $I I P_{3}$ but also a higher $N F$ (denoted here by index $b$ ). Table III shows the analytically estimated results of two such first order filters ( $I a$ and $I b)$ and the corresponding cascaded second order filter (IIab) respectively for the case when the first order filters are designed so that the filter noise figure approach its optimum value. A $20 \mathrm{~dB}$ relative bandwidth of $50 \%$ for each of the two first order filters is assumed which corresponds to $V=0.82$ when $n=2$. 
TABLE III

Analytically estimated results of two first order filters (I) and the corresponding cascaded second order filter (II) respectively for the case when the first order filters are designed so that the filter noise figure approach its optimum value. $\left(\beta_{2}=-0.9 \mathrm{~dB}, V=0.82, n=2, N F_{A}=3 \mathrm{~dB}, O I P_{3, A}\right.$ $=32 \mathrm{dBm})$. (a) Low-noise filter $\left(\beta_{1}=-10 \mathrm{~dB}\right)$. (b) High input IP $P_{3}$ filter: $\left(\beta_{1}=-0.5 \mathrm{~dB}\right)$.

\begin{tabular}{lccccc} 
Filter & $\boldsymbol{B}_{20 d \boldsymbol{B}}$ & $\begin{array}{c}\boldsymbol{G} \\
{[\mathrm{dB}]}\end{array}$ & $\begin{array}{c}\boldsymbol{N F} \\
{[\mathrm{dB}]}\end{array}$ & $\begin{array}{c}\text { OIP3 } \\
{[\mathrm{dBm}]}\end{array}$ & $\begin{array}{c}\boldsymbol{S F D R} \\
{[\mathrm{dB}]}\end{array}$ \\
\hline$I(a)$ & 0.5 & 16.1 & 3.24 & 17.1 & 66.1 \\
$I(b)$ & 0.5 & -3.01 & 10.4 & 17.1 & 74.1 \\
$I I(a b)$ & 0.1 & 13.1 & 3.72 & 12.3 & 64.6 \\
\hline
\end{tabular}

When comparing the results in Table III $(B W=$ $18 \mathrm{MHz}$ ) with the active filter requirements in Table I we note that a cascaded second order recursive active filter can theoretically fulfil the specification given. However, this requires the noise and large-signal properties of the LNA used in the first order filters to be sufficiently good. For example, as shown in Table III, an $O I P_{3, A}$ of at least $32 \mathrm{dBm}$ is required if $N F_{A}$ is not below $3 \mathrm{~dB}$.

\section{CONCLUSION}

Starting from the requirements of typical microwave receivers for future adaptive radar we have theoretically and experimentally investigated the possibilities and the limitations of tunable narrow band recursive active MMIC filters with respect to such on-chip radar receivers. Measurement and simulation results of two different recursive active MMIC filter designs have been compared with the corresponding theoretical results obtained from our analytical filter models. A good agreement between these results was generally found. Using our validated analytical filter models we have also estimated the highest possible filter spurious-free dynamic range at a given relative filter bandwidth for the case when the filter noise figure is approaching that of the low noise amplifier used in the filter. The results indicate that a cascaded second order recursive active MMIC filter with a filter performance adequate for the microwave receivers of future adaptive radar is indeed feasible.

\section{ACKNOWLEDGEMENT}

The Swedish Armed Forces (FM) are acknowledged for the financial support within the SIGA project.

\section{REFERENCES}

[1] L. Pettersson, M. Danestig, and U. Sjöström, "An experimental S-band digital beamforming antenna," IEEE AES Systems Magazine, Nov. 1997.
[2] A. Gustafsson, M. Danestig, R. Malmqvist, and A. Ouacha, "Tuneable S-band filter for on-chip receiver," 1998 Asia-Pacific Microwave Conference, pp. 781-784, Dec. 1998.

[3] M. Danestig, A. Ouacha, B. Carlegrim, T. Tieman, F. van Vliet, and C. Lie Kien Tsoen, "Evaluation of tuneable MMIC filters for radar applications", FOA report, FOA-R—97-00435-408-SE, Feb. 1997.

[4] M. Delmond et al., "Microwave Tunable Active Filter Design in MMIC Technology Using Recursive Concepts," IEEE 1995 Microwave and Millimeter-Wave Monolithic Circuits Symposium, pp. 105-108, May 1995.

[5] M. Danestig, H. Johansson, A. Ouacha, and S. Rudner, "Low Noise Active Recursive MMIC Filters," IEEE MTTS International Symposium Digest, pp. 705-708, June 1997.

[6] H. Ezzedine, M. Delmond, L. Billonet, B. Jarry, and P. Guillon, "Analysis and Optimization of Noise Performance for Various Topologies of Microwave Active Recursive Filters Using Noise Wave Techniques," Microwave and Optical Technology Letters, July 1998.

[7] R. Malmqvist, M. Danestig, S. Rudner, and C. Svensson, "Some Limiting Factors for the Noise Optimization of Recursive Active Microwave Integrated Filters," Microwave and Optical Technology Letters, Aug. 1999.

[8] R. Malmqvist, M. Danestig, S. Rudner, and C. Svensson, "Analysis of Intermodulation and Noise Performance for Recursive Active Microwave Integrated Filters," $29^{\text {th }}$ European Microwave Conference, pp. 60-63, Oct. 1999.

[9] R. Malmqvist, M. Danestig, S. Rudner, and C. Svensson, "Estimation of Spurious-Free Dynamic Range for Recursive Active Microwave Integrated Filters," 1999 Asia-Pacific Microwave Conference, pp. 238-241, Dec. 1999.

[10]M. Danestig, A. Ouacha, C. Lie Kien Tsoen, T. Tieman, and S. Rudner, "Recursive filters employing transmission type phase shifters and novel self-switched time shifters for frequency tuning," $28^{\text {th }}$ European Microwave Conference, pp. 352-357, Oct. 1998. 